\title{
Extremal Zagreb Indices of Graphs with a Given Number of Cut Edges
}

\author{
Shubo Chen · Weijun Liu
}

Received: 13 August 2009 / Revised: 15 October 2012 / Published online: 15 November 2012 (C) The Author(s) 2012. This article is published with open access at Springerlink.com

\begin{abstract}
For a graph, the first Zagreb index $M_{1}$ is equal to the sum of the squares of the degrees of the vertices, and the second Zagreb index $M_{2}$ is equal to the sum of the products of the degrees of pairs of adjacent vertices. Denote by $\mathcal{G}_{n, k}$ the set of graphs with $n$ vertices and $k$ cut edges. In this paper, we showed the types of graphs with the largest and the second largest $M_{1}$ and $M_{2}$ among $\mathcal{G}_{n, k}$.
\end{abstract}

Keywords Zagreb index $\cdot$ Cut edges $\cdot$ Grafting transformation

Mathematics Subject Classification (2000) $05 \mathrm{C} 05 \cdot 92 \mathrm{E} 10 \cdot 05 \mathrm{C} 35$

\section{Introduction}

We use Bondy and Murty [1] for terminology and notions not defined here. Let $G=$ $(V, E)$ be a simple connected graph with $n$ vertices and $m$ edges. For any $v \in V, N(v)$ denotes the neighbors of $v$, and $N_{G}[v]=\{v\} \cup\{u \mid u v \in E(G)\}, d_{G}(v)=|N(v)|$ is the degree of $v$. A leaf is a vertex of degree one and a stem is a vertex adjacent to at least one leaf, pendant edges are edges incident to a leaf and stem, denote it simply as $K_{2}$. The distance $d(x, y)$ from a vertex $x$ to another vertex $y$ is the minimum number of edges in a $x-y$ path. The distance $d_{G}(x, S)$ from a vertex $x$ to the set $S$ is $\min _{y \in S} d(x, y)$. Let $P_{n}$,

\footnotetext{
S. Chen $(\varangle)$

College of Mathematics, Hunan City University, Yiyang 413000, People's Republic of China

e-mail: shubochen@gmail.com

W. Liu

College of Mathematics and Statistics, Central South University,

Changsha 410075, People's Republic of China

e-mail:wjliu6210@126.com
} 
$C_{n}$ and $K_{1, n-1}$ be the path, cycle and the star on $n$ vertices. The cyclomatic number of a connected graph $G$ is defined as $c(G)=m-n+1$. A graph $G$ with $c(G)=k$ is called a $k$ cyclic graph, for $c(G)=0,1$ and 2, we named $G$ as tree, unicyclic graph and bicyclic graph, resp. For any two graphs $G_{1}$ and $G_{2}$, if there exists a common vertex $v$ between them, we denote this graph as $G_{1} v G_{2}$, i.e., the vertex set of $G_{1} v G_{2}$ is $V\left(G_{1}\right) \cup V\left(G_{2}\right), V\left(G_{1}\right) \cap V\left(G_{2}\right)=\{v\}$, and $E\left(G_{1} v G_{2}\right)=E\left(G_{1}\right) \cup E\left(G_{2}\right)$. If there are a copies of graphs $G_{1}, G_{2}, \cdots, G_{l}(l \geq 2)$ with all graphs sharing one common vertex $v$, then, we denote this graph as $G_{1} v G_{2} v \cdots v G_{l}$. If there exists a bridge $u v$ between them such that $u \in V\left(G_{1}\right)$ and $v \in V\left(G_{2}\right)$, we denote this graph as $G_{1} u v G_{2}$. Let $E^{\prime} \subseteq E(G)$, we denote by $G-E^{\prime}$ the subgraph of $G$ obtained by deleting the edges of $E^{\prime} . W \subseteq V(G), G-W$ denotes the subgraph of $G$ obtained by deleting the vertices of $W$ and the edges incident with them.

The first Zagreb index $M_{1}$ and the second Zagreb index $M_{2}$ of $G$ are defined as

$$
\begin{aligned}
& M_{1}(G)=\sum_{x \in V(G)}\left(d_{G}(x)\right)^{2} \\
& M_{2}(G)=\sum_{x y \in E(G)} d_{G}(x) d_{G}(y)
\end{aligned}
$$

The Zagreb indices $M_{1}$ and $M_{2}$ were introduced in [2] and elaborated in [3]. The main properties of $M_{1}$ and $M_{2}$ were summarized in [4,5]. These indices reflect the extent of branching of the molecular carbon-atom skeleton, and can thus be viewed as molecular structure-descriptors [5,6].

Recently, finding the extremal values or bounds for the topological indices of graphs, as well as related problems of characterizing the extremal graphs, attracted the attention of many researchers and many results are obtained (see [3-11]). Nikolić et al. [4] showed that the trees with the smallest and largest $M_{1}$ are the path and the star, respectively. Gutman and Das [7] also showed that the trees with the smallest and largest $M_{2}$ are the path and the star, respectively. In [8] the authors ordered the unicyclic graphs with respect to $M_{1}$ and $M_{2}$. Zhang and Zhang [9] gave the unicyclic graphs with the first three smallest and largest $M_{1}$. Chen and Deng [10] gave the bicyclic graph with the largest $M_{1}$. Deng [11] presented a unified approach to the extremal Zagreb indices for trees, unicyclic graphs and bicyclic graphs. More results in this direction can be found in Refs. [12-23].

Let $\mathcal{G}_{n, k}$ is the set of graphs with $n$ vertices and $k$ cut edges. Let $E^{\prime}=$ $\left\{e_{1}, e_{2}, \cdots, e_{k}\right\}$ is the set of cut edge of $G$, then $E^{\prime}$ can be classified into two kinds, i.e., the pendant edges and non-pendant edges, the number of them are $k^{\prime}, k-k^{\prime}$ resp. The components of $G-\left\{e_{1}, e_{2}, \cdots, e_{k}\right\}$ are 2-edge-connected graphs and isolated vertices. The connected graphs with $k$ cut edges(or vertices) have been considered in many mathematical literatures [24-31]. It is natural to think that, for the $n$ vertex tree, $k=n-1$, and trees with extremal Zagreb indices had been obtained long time ago. For a connected graph on $n$ vertices having the cyclomatic number at least one, the number of its cut edges is most $n-3$, therefore, in our following discussion, we always assume that $G$ has $k$ cut edges with $1 \leq k \leq n-3$. In this paper, we shall investigate the Zagreb indices of $\mathcal{G}_{n, k}$ by introducing some graph grafting operations, and determine the graphs in $\mathcal{G}_{n, k}$ with the largest and the second largest Zagreb indices. 


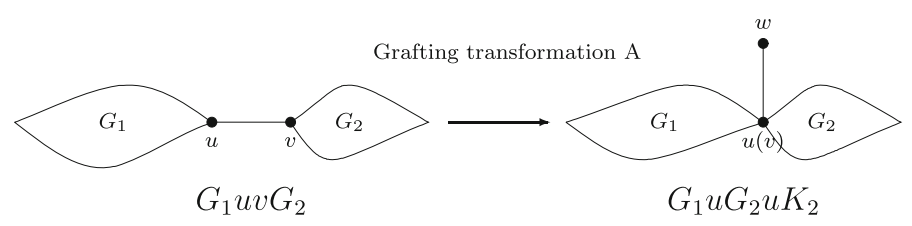

Fig. 1 The grafting transformation A of non-end cut edge $u v$

\section{Grafting Transformations on Graphs with Cut Edges Related to Zagreb Indices}

Before our discussion, we introduce a known proposition, which is useful to our main results.

Proposition 1 Let $G=(V, E)$ is a simple connected graph,

(i) If $e=u v \notin E(G), u, v \in V(G)$. Then, $M_{i}(G)<M_{i}(G+u v)(i=1,2)$;

(ii) If $e \in E(G)$, then, $M_{i}(G)>M_{i}(G-e)(i=1,2)$.

For convenience, we provide some grafting transformations on graphs with cut edges which will increase the Zagreb indices in the following:

Lemma 1 Let $u v$ is a non-pendent cut edge of $G_{1} u v G_{2}, G_{1}$ and $G_{2}$ are 2-edge connected graphs, $G_{1} u v G_{2}$ and $G_{1} u G_{2} u K_{2}$ are two graphs with $\left|V\left(G_{1}\right)\right|,\left|V\left(G_{2}\right)\right| \geq$ 2 , depicted in Fig. 1. Then $M_{1}\left(G_{1} u G_{2} u K_{2}\right)>M_{1}\left(G_{1} u v G_{2}\right), M_{2}\left(G_{1} u G_{2} u K_{2}\right)>$ $M_{2}\left(G_{1} u v G_{2}\right)$.

Proof Let $G=G_{1} u v G_{2}, G^{*}=G_{1} u G_{2} u K_{2}$. By the definition of Zagreb indices, we have

$$
\begin{aligned}
& M_{1}\left(G^{*}\right)-M_{1}(G) \\
& =d_{G^{*}}^{2}(u)+1-d_{G}^{2}(u)-d_{G}^{2}(v) \\
& =\left[d_{G}(u)+d_{G}(v)-1\right]^{2}+1-d_{G}^{2}(u)-d_{G}^{2}(v) \\
& =2\left(d_{G}(u)-1\right)\left(d_{G}(v)-1\right)>0
\end{aligned}
$$

Let $N_{G}(u) \backslash v=\left\{u_{1}, u_{2}, \cdots, u_{r}\right\}(r \geq 2), N_{G}(v) \backslash u=\left\{v_{1}, v_{2}, \cdots, v_{s}\right\}(s \geq 2)$. Then, $d_{G}(u)=r+1, d_{G}(v)=s+1, d_{G^{*}}(u)=r+s+1$. From the assume, we know that $\left\{u_{1}, u_{2}, \cdots, u_{r}\right\} \in V\left(G_{1}\right),\left\{v_{1}, v_{2}, \cdots, u_{s}\right\} \in V\left(G_{2}\right)$.

$$
\begin{aligned}
& \sum_{x \in N_{G^{*}}(u)} d_{G^{*}}(x) d_{G^{*}}(u)-\sum_{x \in N_{G}(u)} d_{G}(x) d_{G}(u)-\sum_{x \in N_{G}(v) \backslash u} d_{G}(x) d_{G}(v) \\
= & (r+s+1)+\sum_{x \in N_{G}(u) \backslash v} d_{G}(x)(r+s+1)+\sum_{x \in N_{G}(v) \backslash u} d_{G}(x)(r+s+1) \\
& -\sum_{x \in N_{G}(u) \backslash v} d_{G}(x)(r+1)-\sum_{x \in N_{G}(u) \backslash v} d_{G}(x)(s+1)-(r+1)(s+1) \\
= & \sum_{i=1}^{r} d_{G}\left(u_{i}\right) s+\sum_{j=1}^{s} d_{G}\left(v_{j}\right) r+r+s+1-(r+1)(s+1) \\
\geq & r s>0 \quad\left(\text { since } d_{G}\left(u_{i}\right) \geq 1\left(d_{G}\left(v_{j}\right) \geq 1 \text { resp. }\right)\right)
\end{aligned}
$$




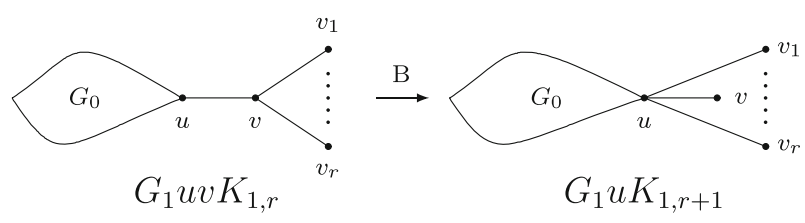

Fig. 2 Grafting transformation B

Fig. 3 The graph $G_{k}^{*}$

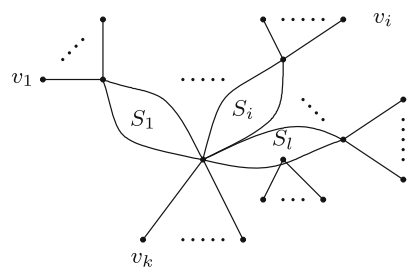

Fig. 4 Grafting transformation $\mathrm{C}$

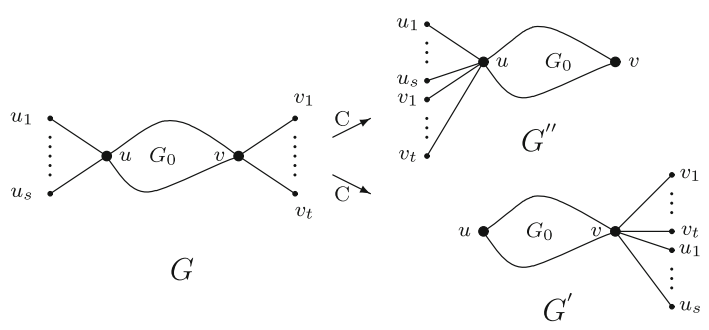

The proof is completed.

Note that the graphs $G_{1} u G_{2} u K_{2}$ has $k$ cut edges, and the pendant vertex increase one, i.e., $w . G_{1}, G_{2}$ are 2-edge-connected graphs as well.

If $u v$ is an non-pendant cut edge of $G_{1} u v K_{1, k}$, as shown in Fig. 2. We shall have

Lemma 2 Let uv is a non-pendant cut edge of $G_{1} u v K_{1, r}, G_{1}$ is a 2-edge connected graphs, $G_{1} u v K_{1, r}$ and $G_{1} u K_{1, r+1}$ are two graphs with $\left|V\left(G_{1}\right)\right| \geq 2$, depicted in Fig. 2. Then $M_{1}\left(G_{1} u K_{1, r+1}\right)>M_{1}\left(G_{1} u v K_{1, r}\right), M_{2}\left(G_{1} u K_{1, r+1}\right)>M_{2}\left(G_{1} u v K_{1, r}\right)$.

Proof It follows from the proof of Lemma 1, the results is obviously.

Remark 1 Repeating the grafting transformation A or B, any cut(non-pendant cut) edge can changed into pendant edge. The graph is shown in Fig. 3, where $S_{i}(1 \leq i \leq l)$ are 2-edge-connected graphs.

Lemma 3 [11] Let $u$ and $v$ be two vertices in $G . u_{1}, u_{2}, \cdots, u_{s}$ are the leaves adjacent to $u, v_{1}, v_{2}, \cdots, v_{t}$ are the leaves adjacent to $v . G^{\prime}=G-\left\{u u_{1}, u u_{2}, \cdots, u u_{s}\right\}+$ $\left\{v u_{1}, v u_{2}, \cdots, v u_{s}\right\}, G^{\prime \prime}=G-\left\{v v_{1}, v v_{2}, \cdots, v v_{t}\right\}+\left\{u v_{1}\right.$, $\left.u v_{2}, \cdots, u v_{t}\right\}$ and $\left|V\left(G_{0}\right)\right| \geq 3$, as shown in Fig. 4. Then, either $M_{i}\left(G^{\prime}\right)>M_{i}(G)$ or $M_{i}\left(G^{\prime \prime}\right)>M_{i}(G), i=1,2$.

Remark 2 Repeating grafting transformation $\mathrm{C}$, all the pendant edges are attached to the same vertex. 

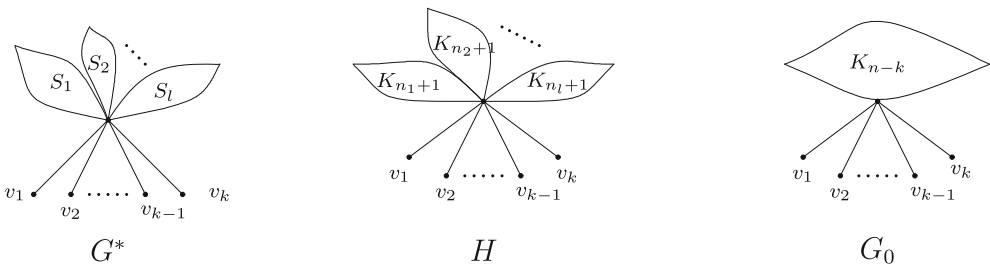

Fig. 5 Three simple graphs with $k$ cut edges

\section{Graphs in $\mathcal{G}_{n, k}$ with the Largest, the Second Largest Zagreb Indices}

In this section we shall get graphs in $\mathcal{G}_{n, k}$ with the largest, the second largest Zagreb indices.

\subsection{Graphs in $\mathcal{G}_{n, k}$ with the Largest Zagreb Indices}

In this section, we discuss graphs in $\mathcal{G}_{n, k}$ with the largest $M_{1}, M_{2}$.

Theorem 1 Of all the connected graphs in $\mathcal{G}_{n, k}(1 \leq k \leq n-3)$, the maximum $M_{1}$, $M_{2}$ values are obtained uniquely at $K_{n}^{k}$. Where $K_{n}^{k}$ is a graph obtained by joining $k$ independent vertices to one vertex of $K_{n-k}$.

Proof Firstly, from above Lemmas we present the graphs in $\mathcal{G}_{n, k}$ the upper bounds, respectively, for $M_{1}, M_{2}$. As described above, we obtained graphs in the following (see Fig. 5) will achieve the upper bounds with respect to $M_{1}, M_{2}$.

Nextly, we consider three Claim as follows.

Claim 1 If a graph $G \in \mathcal{G}_{n, k}$, then $M_{i}(G) \leq M_{i}\left(G^{*}\right)$ for $i=1,2$.

Proof of Claim 1 Suppose that $G \in \mathcal{G}_{n, k}$ and $G \supsetneqq G^{*}$, then by the proof of Lemma 1, Lemma 2 and Lemma 3, we know $M_{i}(G)<M_{i}\left(G^{*}\right)$ for $i=1$, 2. Obviously, when $G \cong G^{*}, M_{i}(G)=M_{i}\left(G^{*}\right)$ for $i=1,2$, which completes the proof.

Claim 2 For the graph $G^{*}$, we have $M_{i}\left(G^{*}\right) \leq M_{i}(H)(i=1,2)$, and the equalities hold if and only $G^{*} \cong H$.

Proof of Claim 2 Let $\left\{e_{1}, e_{2} . \cdots, e_{k}\right\}$ be the set of the cut edges of $G \in \mathcal{G}_{n, k}$. Note that by Proposition 1, if we add an edge not belong to $G$, then, we have $M_{i}(G+e)>$ $M_{i}(G)$ for $i=1,2$. For the 2-edge-connected graph $S_{i}(i=1,2, \cdots, l)$, we add edges in them, changing them into complete sub-graphs $K_{n_{i}+1}(i=1,2, \cdots, l)$, thus the graphs $G^{*}$ is changed into the graph $H$, and $H$ is still possesses $k$ cut edges, i.e., $H \in G \in \mathcal{G}_{n, k}, G^{*} \leq H$. By the Proposition, if $G^{*}<H$, then $M_{i}\left(G^{*}\right)<M_{i}(H)$ $(i=1,2)$, else, $M_{i}\left(G^{*}\right)=M_{i}(H)(i=1,2)$ iff $G^{*} \cong H$.

This completes the proof of Claim 2.

Claim $3 M_{i}(H) \leq M_{i}\left(G_{0}\right)(i=1,2)$, the equalities hold if and only $H \cong G_{0}$, i.e., $l=1$.

Proof of Claim 3

If we add edges between the every two vertices of completed sub-graphs $K_{n_{i}+1}$ $(i=1,2, \cdots, l)$ of $H, H$ will be changed into the graph $G_{0}$, and $G_{0}$ is still possesses 

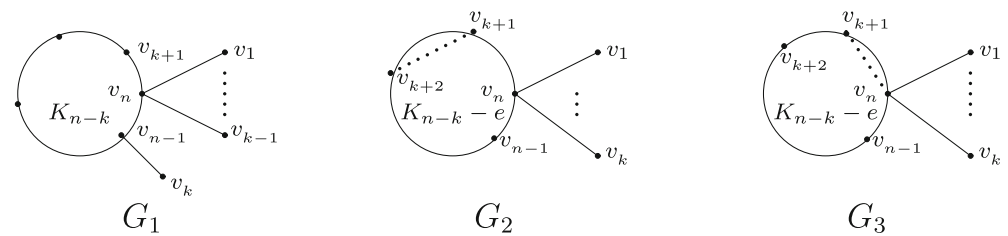

Fig. 6 Three simple connected graphs with cut edges

$k$ cut edges, i.e., $G_{0} \in G \in \mathcal{G}_{n, k}$, and $H<G_{0}$. Thus, by Proposition 1, we have $M_{i}(H) \leq M_{i}\left(G_{0}\right)(i=1,2)$, the equalities hold if and only $H \cong G_{0}$, i.e., $l=1$.

This completes the proof of Claim 3 .

Combine above Claims, theorem holds.

\subsection{Graphs in $\mathcal{G}_{n, k}$ with the Second Largest Zagreb Indices}

In this section, we shall determine graphs in $\mathcal{G}_{n, k}$ with the second largest $M_{1}, M_{2}$, respectively.

Firstly, we characterize the three graphs will achieve the upper bounds on $M_{1}, M_{2}$.

Theorem 2 For an arbitrary graph $G \in \mathcal{G}_{n, k}(1 \leq k \leq n-3)$, and $G ¥ G_{0}$, we have

(i) If $1 \leq k \leq 4$, then $M_{1}(G) \leq M_{1}\left(G_{1}\right)$, the equalities hold if and only if $G \cong G_{1}$;

(ii) If $k>4$, and $n \leq\left[\frac{3}{2} k+1\right]$, then $M_{1}(G) \leq M_{1}\left(G_{1}\right)$, the equalities hold if and only if $G \cong G_{2}$;

(iii) If $k>4$, and $n>\left[\frac{3}{2} k+1\right]$, then $M_{1}(G)<M_{1}\left(G_{1}\right)$;

(iv) $M_{2}(G) \leq M_{2}\left(G_{1}\right)$, the equalities hold if and only if $G \cong G_{1}$.

Proof Firstly, we shall prove that the graph $G \in \mathcal{G}_{n, k}(1 \leq k \leq n-3)$, and $G ¥ G_{0}$ attain the maximum $M_{i}(G)(i=1,2)$, then $G$ must be one of the graph $G_{1}, G_{2}$ and $G_{3}$ depicted in Fig. 6.

Follows the remark 1 , we get the graph $G_{k^{\prime \prime}}^{*}$, and $M_{i}\left(G_{k^{\prime \prime}}^{*}\right) \geq M_{i}(G)(i=1,2)$, where $k^{\prime \prime}$ is the number of non-pendant vertices connected the cut edges. From the structure of $G_{k^{\prime \prime}}^{*}$, we divide our discussion into two cases according to parameters $l$ and $k^{\prime \prime}$.

Case 1 When $l=1$, there are two subcases in the following:

Subcase 1.1 If $k^{\prime \prime}=1$.

In this case, we adding edges to the vertices of the 2-edge-connected subgraph $S_{1}$, thus, $S_{1}$ can be changed into the graph $G_{2}$ or $G_{3}$, see Fig. 7. Adding one edge again to $G_{2}$ or $G_{3}$, they can be changed into $G_{0}$. By the proposition 1, we have

(i) $M_{i}(G) \leq M_{i}\left(G_{k^{\prime \prime}}^{*}\right) \leq M_{i}\left(G_{2}\right) \leq M_{i}\left(G_{0}\right)(i=1,2)$; or

(ii) $M_{i}(G) \leq M_{i}\left(G_{k^{\prime \prime}}^{*}\right) \leq M_{i}\left(G_{3}\right) \leq M_{i}\left(G_{0}\right)(i=1,2)$.

Subcase 1.2 If $k^{\prime \prime} \geq 2$.

In this case, at first, we adding edges to the vertices of the 2-edge-connected subgraph $S_{1}$, thus, $S_{1}$ can be changed into the graph $K_{n-k}$, see Fig. 8, denote the 
Fig. 7 The transformation of graph $G_{k^{\prime \prime}}^{*}$ when $l=1, k^{\prime \prime}=1$
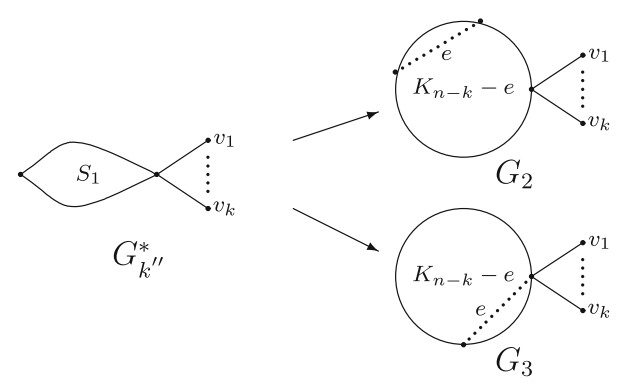

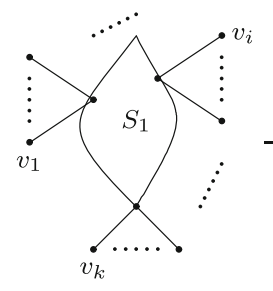

$G_{k^{\prime \prime}}^{*}$

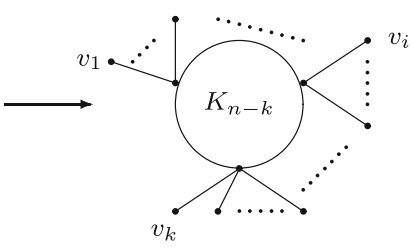

$H_{1}$

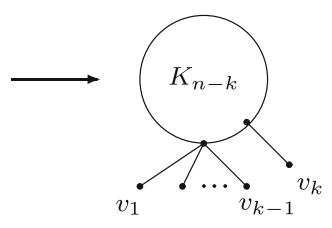

$G_{1}$

Fig. 8 The transformation of graph $G_{k^{\prime \prime}}^{*}$ when $l=1, k^{\prime \prime} \geq 2$
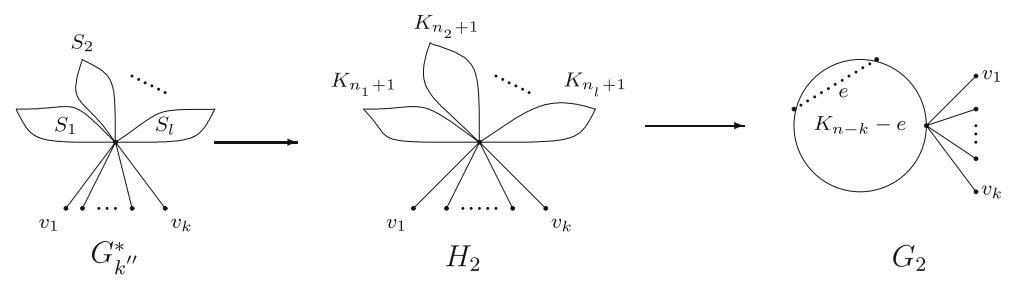

Fig. 9 The transformation of $G_{k^{\prime \prime}}^{*}$ when $l \geq 2, k^{\prime \prime}=1$

graph as $H_{1}$. By the proposition 1 , we have $M_{i}(H) \geq M_{i}\left(G_{k^{\prime \prime}}^{*}\right)(i=1,2)$. In the next, repeating graft transformation $\mathrm{C}$ on $H$, we can get the graph $G_{1}$. Obviously, we only graft one edge of $G_{1}, G_{1}$ will change into $G_{0}$. By Lemma 3 , we have $M_{i}(G) \leq M_{i}\left(H_{1}\right) \leq M_{i}\left(G_{1}\right) \leq M_{i}\left(G_{0}\right)(i=1,2)$.

Case 2 When $l \geq 2$, similar to the Case 1, there are two subcases in the following:

Subcase 2.1

If $k^{\prime \prime}=1$.

Firstly, we add edges in the 2-edge-connected subgraphs $S_{i}(i=1,2, \cdots, l)$ of $G_{k^{\prime \prime}}^{*}$, and we get complete graphs $K_{i+1}(i=1,2, \cdots, l)$, which composed the graph $H_{2}$ ( see Fig. 9). By the proposition 1 , we have $M_{i}\left(H_{2}\right) \geq M_{i}\left(G_{k^{\prime \prime}}^{*}\right)(i=1,2)$. Then, adding edges between $K_{i+1}(i=1,2, \cdots, l)$, we shall get the graph $G_{2}$ ( see Fig. 9), and if we add one edge again to $G_{2}$, it can be changed into the graph $G_{0}$. By proposition 1 , we know that $M_{i}\left(G_{k^{\prime \prime}}^{*}\right) \leq M_{i}\left(H_{2}\right) \leq M_{i}\left(G_{2}\right) \leq M_{i}\left(G_{0}\right)(i=1,2)$. 

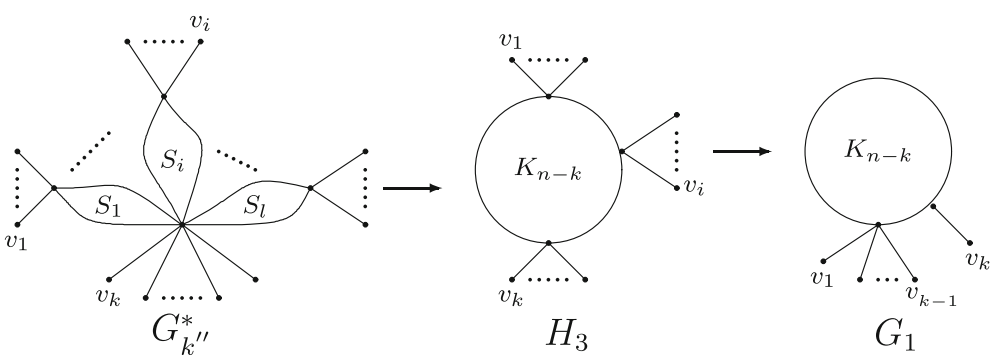

Fig. 10 The transformation of $G_{k^{\prime \prime}}^{*}$ when $l \geq 2, k \geq 2$

Subcase 2.2. If $k^{\prime \prime} \geq 2$.

Similar to the method of subcase 2.1, firstly, we add edges in the 2-edge-connected subgraphs $S_{i}(i=1,2, \cdots, l)$ of $G_{k^{\prime \prime}}^{*}$, and we get complete graphs $K_{i+1}(i=$ $1,2, \cdots, l)$, Then, adding edges between $K_{i+1}(i=1,2, \cdots, l)$, we shall get the graph $H_{3}$ ( see Fig. 10). At last, applying grafting transformation $\mathrm{C}$ on $\mathrm{H}_{3}$, we shall get the graph $G_{1}$, and if we add one edge again to $G_{1}$, it can be changed into the graph $G_{0}$. By proposition 1 and Lemma 3 , we know that $M_{i}\left(G_{k^{\prime \prime}}^{*}\right) \leq M_{i}\left(H_{3}\right) \leq M_{i}\left(G_{1}\right) \leq$ $M_{i}\left(G_{0}\right)(i=1,2)$.

From above operations, it is sufficient to see that one of the graphs $G_{1}, G_{2}$ and $G_{3}$ attains the second largest $M_{i}(i=1,2)$. In our following work, we only need to compare the Zagreb indices $M_{i}(i=1,2)$ of $G_{1}, G_{2}$ and $G_{3}$.

By simple calculation, we have:

$$
\begin{aligned}
& M_{1}\left(G_{1}\right)=(n-2)^{2}+(n-k)^{2}+(n-k-2)(n-k-1)^{2}+k \\
& M_{1}\left(G_{2}\right)=(n-1)^{2}+2(n-k-2)^{2}+(n-k-3)(n-k-1)^{2}+k \\
& M_{1}\left(G_{3}\right)=(n-2)^{2}+(n-k-2)^{2}+(n-k-2)(n-k-1)^{2}+k
\end{aligned}
$$

$$
\begin{gathered}
M_{2}\left(G_{1}\right)=(n-2)\left[(n-k)^{2}-3 n+4 k+1\right]+(n+1)(n-k)+(n-k)^{3} \\
-3(n-k)^{2}+(n-k-1)^{2}\left[\frac{1}{2}(n-k)^{2}-\frac{5}{2}(n-k)+3\right] ; \\
M_{2}\left(G_{2}\right)=(n-1)\left[(n-k)^{2}-2 n+3 k-1\right]+2(n-k-1)(n-k-2) \\
(n-k-3)+(n-k-1)^{2}\left[\frac{1}{2}(n-k)^{2}-\frac{7}{2}(n-k)+6\right] ; \\
M_{2}\left(G_{3}\right)=k(n-2)+(n-k-2)(n-k-1)(2 n-k-4) \\
+(n-k-1)^{2}\left[\frac{1}{2}(n-k)^{2}-\frac{5}{2}(n-k)+3\right] .
\end{gathered}
$$


Therefore

$$
\begin{aligned}
& \Delta_{1}=M_{1}\left(G_{1}\right)-M_{1}\left(G_{2}\right)=4\left(n-\frac{3}{2} k-1\right) \\
& \Delta_{2}=M_{1}\left(G_{2}\right)-M_{1}\left(G_{3}\right)=2 k>0 \\
& \Delta_{3}=M_{2}\left(G_{1}\right)-M_{2}\left(G_{2}\right)=3(n-k)^{2}-8(n-k)+k+6>0 \\
& \Delta_{4}=M_{2}\left(G_{2}\right)-M_{2}\left(G_{3}\right)=k(n-2-k)>0
\end{aligned}
$$

As pointed out before, we have $1 \leq k \leq n-3$.

Let $k+3=\frac{3}{2} k+1$, then $k=4$. Therefore,

(i) $\Delta_{1} \geq 0$, if $1 \leq k \leq 4$. otherwise

(ii) $\Delta_{1}<0$, if $k>4$ and $n \leq\left[\frac{3}{2} k+1\right]$, where $[x]$ stands for the integer part of $x$, or else

(iii) $\Delta_{1}>0$, if $k>4$ and $n>\left[\frac{3}{2} k+1\right]$.

The proof of theorem is completed.

Acknowledgements Projects Supported by the National Natural Science Foundation of China (11271208) and Hunan Provincial Natural Science Foundation (12JJ3005)

Open Access This article is distributed under the terms of the Creative Commons Attribution License which permits any use, distribution, and reproduction in any medium, provided the original author(s) and the source are credited.

\section{References}

1. Bondy, J.A., Murty, U.S.R.: Graph Theory with Applications. Macmillan, New York (1976)

2. Gutman, I., Trinajstić, N.: Graph theory and molecular orbitals. Total $\pi$-electron energy of alternant hydrocarbons. Chem. Phys. Lett. 17, 535-538 (1972)

3. Gutman, I., Ruščić, B., Trinajstić, N., Wilcox, C.F.: Graph theory and molecular orbitals. XII. Acyclic Polyenes J. Chem. Phys. 62(195), 3399-3405

4. Nikolić, S., Kovačević, G., Miličević, A., Trinajstić, N.: The Zagreb indices 30 years after. Croat. Chem. Acta 76, 113-124 (2003)

5. Gutman, I., Das, K.C.: The first Zagreb index 30 years after. MATCH Commun. Math. Comput. Chem 50, 83-92 (2004)

6. Balaban, A.T., Motoc, I., Bonchev, D., Mekenyan, O.: Topological indices for structure-activity corrections. Topics Curr. Chem 114, 21-55 (1983)

7. Gutman, I., Das, K.C.: The first Zagreb index 30 years after. MATCH Commun. Math. Comput. Chem 50, 83-92 (2004)

8. Xia, F., Chen, S.: Ordering unicyclic graphs with respect to Zagreb indices. MATCH Commun. Math. Comput. Chem 58, 663-673 (2007)

9. Zhang, H., Zhang, S.: Uncyclic graphs with the first three smallest and largest first general Zagreb index. MATCH Commun. Math. Comput. Chem. 55, 427-438 (2006)

10. Chen, S., Deng, H.: Extremal $(n, n+1)$-graphs with respected to zeroth-order Randic index. J. Math. Chem. 42, 555-564 (2007)

11. Deng, H.: A unified approach to the extremal Zagreb indices for trees, unicyclic graphs and bicyclic graphs. MATCH Commun. Math. Comput. Chem 57, 597-616 (2007)

12. Zhou, B.: Zagreb indices. MATCH Commun. Math. Comput. Chem 52, 113-118 (2004)

13. Liu, B., Gutman, I.: Upper bounds for Zagreb indices of connected graphs. MATCH Commun. Math. Comput. Chem. 55, 439-446 (2006)

14. Zhou, B., Gutman, I.: Further properties of Zagreb indices. MATCH Commun. Math. Comput. Chem 54, 233-239 (2005) 
15. Vukičević, D., Trinajstić, N.: On the discriminatory power of the Zagreb indices for molecular graphs. MATCH Commun. Math. Comput. Chem. 53, 111-138 (2005)

16. Gutman, I., Furtula, B., Toropov, A.A., Toropov, A.P.: The grpah of atomic orbitals and its basic properties. 2. Zagreb indices. MATCH Commun. Math. Comput. Chem 53, 111-138 (2005)

17. Nikolić, S., Tolić, I.M., Trinajstić, N., Baučić, I.: On the Zagreb indices as complexity indices. Croat. Chem. Acta 73, 909-921 (2000)

18. Das, K., Gutman, I., Zhou, B.: New upper bounds on Zagreb indices. J. Math. Chem 46, 514-521 (2009)

19. $\mathrm{Xu}, \mathrm{K}$.: The Zagreb indices of graphs with a given clique number. Appl. Math. Lett 24, 1026-1030 (2011)

20. Behtoei, A., Jannesari, M., Taeri, B.: Maximum Zagreb index, minimum hyper-Wiener index and graph connectivity. Appl. Math. Lett. 22, 1571-1576 (2009)

21. Das, K.: On comparing Zagreb indices of graphs. MATCH Commun. Math. Comput. Chem. 63, 433-440 (2010)

22. Hfath-Tabar, G.H.: Old and new Zagreb indices of graphs. MATCH Commun. Math. Comput. Chem. 65, 79-84 (2011)

23. Ashrafi, A.R., DoŠlić, T., Hamzeh, A.: Extremal graphs with respect to the Zagreb coindices. MATCH Commun. Math. Comput. Chem 65, 85-92 (2011)

24. Zhao, Q., Li, S.C.: On the maximum Zagreb indices of graphs with $k$ cut vertices. Acta Appl. Math. 111, 93-106 (2010)

25. Li, S.C., Zhao, Q.: Sharp upper bounds on Zagreb indices of bicyclic graphs with a given matching number. Math. Comput. Model. 54, 2869-2879 (2011)

26. Liu, H., Lu, M., Tian, F.: On the spectral radius of graphs with cut edges. Linear Algebra Appl. 389, 139-145 (2004)

27. Wu, Y.R., He, S., Shu, J.L.: Largest spectral radius among graphs with cut edges. J. East China Norm. Univ. Nat. Sci. Ed. 3, 67-74 (2007)

28. Pepper, R., Henry, G., Sexton, D.: Cut edges and independence number. MATCH Commun. Math. Comput. Chem 56, 403-408 (2006)

29. Deng, H.: On the Minimum Kirchhoff index of graphs with a given cut edges. MATCH Commun. Math. Comput. Chem. 63, 171-180 (2110)

30. Balakrishnan, R., Sridharan, N., Viswanathan Iyer, K.: Wiener index of graphs with more than one cut vertex. Appl. Math. Lett. 21, 922-927 (2008)

31. Berman, A., Zhang, X.D.: On the spectral radius of graphs with cut vertices. J. Comb. Theory, Ser. B 83, 233-240 (2001) 